Fecha de recepción: abril 2018 Fecha de aceptación: noviembre 2018 Versión final: diciembre 2020

\section{Más allá del producto: un abordaje local sobre el Diseño de Producto-Sistema- Servicio para la sustentabilidad y Tecnologías de Inclusión Social}

Mariana González Insua *

Resumen: Inicialmente, el abordaje del Diseño Sustentable en Argentina estuvo dominado por las prácticas del reciclaje, resolviendo problemas hacia el fin de vida de los productos. Posteriormente, la noción de Ciclo de Vida permitió ampliar los límites de análisis para dar cuenta del impacto de las decisiones de diseño en todas las etapas. Si bien ambos abordajes han sido de utilidad, en el ámbito de la gestión del diseño estas miradas resultan limitadas, porque en la práctica convergen algo más que objetos: los actores, las organizaciones y sus múltiples relaciones. Abonando a los criterios de sustentabilidad, cabe preguntarse cuáles son las herramientas y metodologías necesarias para un abordaje integral de los problemas en la gestión de Diseño. Herramientas que permitan ordenar la información y sirvan también como puente para el planteo conjunto de los problemas con los actores en el territorio.

El enfoque PSS abandona la concepción tradicional de diseño, desarrollo y fabricación de productos para situarse en soluciones en donde la generación de valor se separa del consumo de recursos y energía (Vezzoli et al, 2014). De esta manera las innovaciones están puestas en el diseño de servicios y relaciones entre los actores., a partir de su aplicación práctica en un caso de estudio en la fase de análisis estratégico.

Las Tecnologías para la Inclusión Social (TIS) en la misma línea, se orientan a la generación de capacidades de resolución de problemas sistémicos, y a la generación de dinámicas locales de producción, cambio tecnológico e innovación socio-técnicamente adecuadas que beneficien a todos (Thomas et al, 2012).

En esta línea, el presente artículo revisa las nociones y herramientas propuestas por dos marcos teóricos, por un lado el enfoque Producto-Sistema-Servicio (PSS) y por el otro las Tecnologías de Inclusión Social con el objetivo de aportar a la perspectiva de los abordajes de sustentabilidad urbana a partir de una aproximación interdisciplinar. Se toma como caso de estudio el análisis del Circuito Formal de recuperación de residuos sólidos urbanos de Mar del Plata, analizando por un lado la "Situación-Problema" que determina el estado del sistema actual desde el marco de las Tecnologías de Inclusión Social, y por el otro lado la aplicación del Mapa sistémico como herramienta metodológica complementaria en el análisis.

En la gestión estratégica y aproximaciones sistémicas, es fundamental comprender las problemáticas, expectativas, las capacidades de negociación y alineación de los actores así como a organización de las mismas dentro del territorio. La aproximación metodológica ha resultado complementaria permitiendo a través del marco de TIS la visibilización de la red heterogénea de actores de tienen relevancia significativa a nivel social, económico, político y cultural, y como cada Grupo Social Relevante perteneciente a esta red concibe las problemáticas en relación al objeto de estudio. 
Asimismo el Mapa Sistémico como herramienta de PSS aplicada en el análisis estratégico, contribuye a ordenar y visualizar las relaciones entre los actores, artefactos, tecnologías e instituciones, así como los flujos información, material, económicos, etc.

Palabras clave: Producto-Sistema-Servicio - Tecnologías de Inclusión Social - Residuos Sólidos Urbanos Reciclables - Diseño para la Sustentabilidad - Sustentabilidad Urbana.

[Resúmenes en inglés y portugués en las páginas 107-109]

\begin{abstract}
${ }^{(*)}$ Mariana González Insua. CONICET. Instituto del Hábitat y del Ambiente (IHAM). Facultad de Arquitectura Urbanismo y Diseño. Universidad Nacional de Mar del Plata. Diseñadora Industrial con orientación en productos de la Facultad de Arquitectura Urbanismo y Diseño (FAUD), Universidad Nacional de Mar del Plata (UNMdP). Coordinadora de la red LeNS (Learning Network on Sustainability) en la FAUD UNMDP. Actualmente cursando estudios de postgrado - Magister en Gestión Ambiental del Desarrollo Urbano en el Instituto del Hábitat y del Ambiente (IHAm) de la FAUD-UNMdP. Becaria Interna doctoral (2014-2019) del Consejo Nacional de Investigaciones Científicas y Técnicas (CONICET) con el proyecto de investigación "Desarrollo de estrategias de revalorización de materiales provenientes de los Residuos Sólidos Urbanos de la ciudad de Mar del Plata y su vinculación con la Sustentabilidad Urbana" que se desarrolla en el IHAM-FAUD-UNMDP. Integrante del proyecto de investigación (2015-2016) "Midiendo la sustentabilidad urbana. Tres escalas de análisis y aplicación: urbana-intraurbana; local-región y nacional" (IHAM-FAUD-UNMdP. Ayudante Adscripto de la Catedra de Taller de Diseño I B de la FAUD UNMdP. gonzalezinsuamariana@gmail.com.
\end{abstract}

\title{
Introducción
}

El enfoque Producto-Sistema-Servicio (PSS) abandona la concepción tradicional de diseño, desarrollo y fabricación de productos para situarse en soluciones en las que la generación de valor se separa del consumo de recursos y energía (Vezzoli et al, 2014). De esta manera las innovaciones están puestas en el diseño de servicios y en las relaciones entre los actores.

La definición de un PSS Sustentable propuesto por el proyecto LeNS, en el marco de economías distribuidas, hace referencia a un modelo de oferta que proporcione una mezcla integrada de productos y servicios que juntos puedan satisfacer una demanda particular de los clientes (entregar una "unidad de satisfacción”) basada en interacciones innovadoras entre las partes interesadas del sistema de producción de valor (sistema de satisfacción) económico y competitivo de los proveedores que busca continuamente soluciones ambientales y socio-éticamente beneficiosas (Vezolli et al, 2014).

La UNEP $(2002,2009)$ puntualiza tres tipologías de PSS en relación a su potencial para desarrollarse con resultados que contribuyan a la eco-eficiencia del sistema: 1) el PSS orien- 
tado a producto; 2) orientado a resultados y 3 ) orientado al uso. Estos tres tipos de aproximaciones tienen el potencial de generar soluciones a través del análisis de las interacciones entre los actores involucrados, ya sea agregando valor al ciclo de vida del producto, ofreciendo resultados a los clientes o facilitando plataformas a los mismos. Sin embargo, no todas las innovaciones en los PSS son eco-eficientes o contribuyen a la sustentabilidad del sistema, sino que deben ser diseñadas específicamente para tal fin (Vezolli et al, 2014). Las principales herramientas en relación a PSS - Diseño para la Sustentabilidad, están relacionadas con tres elementos claves (Vezolli et al, 2014): 1) el enfoque de sistema de satisfacción, en el cual se diseña la satisfacción de una demanda particular (unidad de satisfacción) y los productos o servicios relacionados; 2) la configuración de los actores intervinientes, es decir el diseño de las interacciones de los actores de un sistema que responde a una demanda de satisfacción y 3 ) el enfoque de la sustentabilidad del sistema, es decir el diseño de las configuraciones de los actores con el objetivo de generar soluciones cohesivas de carácter eco eficientes, que contribuyan a la equidad social y al desarrollo local.

El diseño de una demanda particular de satisfacción involucra un cambio de pensamiento entre diseñar para "satisfacer" una necesidad o diseñar para "tener un producto que satisfaga” esa necesidad (Ehrenfeld, 2008). Asimismo implica por un lado una aproximación más amplia, ya que incorpora en el análisis más actores, más productos y servicios, y por el otro, reducir el enfoque en base a la satisfacción de una unidad específica (Vezolli et al, 2014).

El diseño de las interacciones de los actores, se orienta a la generación de interacciones y alianzas entre aquellos que resultan relevantes y que contribuyan a responder a la demanda de satisfacción. Por lo tanto diseñar las interacciones implica comprender cuales son los componentes del sistema (actores y características en relación a la demanda de satisfacción) y cuales con las posibles relaciones o conexiones entre (Vezolli et al, 2014). Los métodos y herramientas para de PSS apuntan a contribuir en el desarrollo de 3 objetivos específicos: 1) la evaluación del sistema existencia y el establecimiento de las prioridades en relación a la sustentabilidad del mismo; 2) el desarrollo de sistemas innovadores y 3) la comparación del existente con el sistema innovador a partir de la visualización y comprobación de la mejora en mismo.

Con el objetivo de desarrollar y testear métodos y herramientas para el diseño de sistemas se han desarrollado diferentes proyectos, financiados principalmente por la Unión Europea y la UNEP, entre los cuales se encuentran SusHouse ${ }^{1}, \mathrm{ProSecCo}^{2}, \mathrm{HiCS}^{3}, \mathrm{MEPSS}^{4}$, SusProNet ${ }^{5}$, MSDS and Design for Sustainability ${ }^{6}$ Las Metodologías de Diseño para la Sustentabilidad o Methodology for System Design for Sustainability (MSDS) son desarrolladas por el proyecto LeNS (Learning Network on Sustanability).

La metodología MSDS consiste en 4 fases: 1) análisis estratégico, 2) exploración de oportunidades, 3) diseño de conceptos sistémicos y 4) diseño e ingeniería del sistema conceptualizado. En algunos casos también se considera una quinta etapa que involucra la comunicación. Las herramientas de diseño propuestas por la metodología de Sistemas de Diseño para la Sustentabilidad o MSDS, se pueden clasificar en dos grupos, por un lado las herramientas para visualización sistémica y por el otro las que tienen como objetivo estimular y acompañar el proceso de generación de ideas y estrategias sistémicas. 
Por otro lado, en el abordaje a la sustentabilidad urbana de carácter inclusivo desde una perspectiva socio-técnica, las Tecnologías para la Inclusión Social (TIS) se orientan a la generación de capacidades de resolución de problemas sistémicos, y a la generación de dinámicas locales de producción, cambio tecnológico e innovación socio-técnicamente adecuadas que beneficien a todos (Thomas et al, 2012).

Thomas (2009) define Tecnología Social como una forma de diseñar, desarrollar, implementar y gestionar tecnología orientada a resolver problemas sociales y ambientales, generando dinámicas sociales y económicas de inclusión social y de desarrollo sustentable que alcanzan un amplio abanico de producciones de tecnologías de producto, proceso y organización. Es así que los procesos de desarrollo de Tecnologías Sociales ponen foco en los actores relevantes de la región entre los que se encuentran los movimientos sociales, cooperativas populares, ONGs, unidades públicas de $\mathrm{I}+\mathrm{D}$, divisiones gubernamentales y organismos descentralizados, empresas públicas y, en menor medida, las empresas privadas. El mencionado autor sostiene, asimismo, que la aproximación al análisis de las problemáticas de la pobreza, la exclusión y el subdesarrollo no puede ser analizada sin tener en cuenta la dimensión tecnológica (Thomas, 2009). Por lo tanto, la inclusión de comunidades y grupos sociales dependerá, probablemente, de la capacidad local de generación de soluciones tecno-productivas tanto adecuadas cuanto eficaces.

En esta línea, el funcionamiento o no funcionamiento ${ }^{7}$ de estas tecnologías refleja los problemas existentes en la concepción de los artefactos y sistemas, que no pueden ser explicados simplemente por motivos de "no adopción de artefactos "técnicamente bien diseñados" o " problemas de implementación” sino más bien debido a problemas relacionados con la conceptualización de las tecnologías y la derivación entre las disputas, presiones, resistencias, negociaciones y convergencias que van conformando el ensamble de actores, conocimientos y artefacto (Thomas, 2009).

En el análisis de las dinámicas de desarrollo inclusivo sustentable, comprender la configuración socio-técnica es clave, ya que las estrategias y acciones orientadas a dinamizar procesos se desenvuelven dentro de múltiples dinámicas y/o trayectorias que son contenidas dentro de dinámicas socio-técnicas ${ }^{8}$. Por lo tanto, identificar y analizar funcionamientos que operan en sincronía o en tensión en el territorio, es un factor clave para comprender la situación actual y por lo tanto posibilitar la materializaron de estrategias a nivel sistémico que contribuyan a una situación más sustentable.

Dado que, tanto las alianzas, como las dinámicas y trayectorias responden a diferentes criterios de recorte topológico, metodológicamente hablando, es la pregunta-problema de investigación la que define el alcance de las relaciones que son mapeadas y analizadas y delimitan el sistema. Es así que la construcción de la Situación-Problema como primer objetivo desde el marco de las TIS en el análisis estratégico, recaba la mirada de los problemas de los múltiples grupos sociales en el territorio desde una flexibilidad interpretativa. Dicho de otra manera, identifica cuales son los problemas enunciados por las comunidades, las instituciones, los actores que habitan en el territorio determinado.

De lo planteado anteriormente se desprende el interrogante sobre la complementariedad de las disciplinas de PSS y TIS y sus respectivas herramientas en el abordaje integral y sistémico de problemas abiertos y complejos que involucran la sustentabilidad a nivel urbano. 
El presente trabajo pretende aportar a la perspectiva de los abordajes de sustentabilidad urbana a partir de una aproximación interdisciplinar que involucra los marcos teóricos y herramientas metodológicas de Producto-Sistema-Servicio y Tecnologías de Inclusión Social en la fase de análisis estratégico.

Lo presentado en este trabajo forma parte de un proyecto de investigación más amplio que tiene por objetivo el desarrollo de estrategias de valorización de residuos sólidos urbanos en la ciudad de Mar del Plata. El mismo parte del análisis de las dinámicas socio-técnicas en el territorio con el objetivo de comprender la situación actual del Circuito Formal de recuperación de residuos sólidos urbanos. Se apoya en el análisis socio-técnico y en las herramientas metodológicas de PSS para realizar el análisis estratégico y detectar los puntos críticos a intervenir con el objetivo de contribuir a la sustentabilidad urbana en Mar del Plata.

Metodológicamente se toma como caso de estudio el análisis del Circuito Formal de recuperación de residuos sólidos urbanos de Mar del Plata, analizando por un lado la "Situación-Problema" que determina el estado del sistema actual desde el marco de las Tecnologías de Inclusión Social, y por el otro lado la aplicación del Mapa sistémico como herramienta metodológica complementaria en el análisis.

Se realiza observación participante y entrevistas a profundidad a actores relevantes en el territorio, integrantes de la Cooperativa de Trabajo CURA Ltda., en el área de Desarrollo Social del Ente de Obras y Servicios Urbanos (ENOSUR) de la MGP y en el equipo de Vinculación Socio Productiva (Vi.So.Pro) de la Universidad Nacional de Mar del Plata. Se realiza una revisión de antecedentes sobre los circuitos de recuperación en la ciudad de Mar del Plata y el proceso de conformación de CURA Ltda. en relación a la implementación de la GIRSU. Se analiza el circuito de acumulación de reciclables y sus características a partir de investigaciones previas (Paiva, 2005, 2008, 2009; Schamber et al, 2006; Calo, 2009; Carenzo, 2013; Barabino, 2014, entre otros) focalizando el análisis en los actores localizados en la ciudad de Mar del Plata. Asimismo se trabaja con fuentes de información secundaria tales como los informes anuales ambientales elaborados por municipio de General Pueyrredón.

Las preguntas principales que guían este análisis son ¿Cuáles son los principales actores? ¿Cuál es la relación entre los actores o grupos sociales relevantes?, ¿Cuáles son las problemáticas que se identifican como nudos críticos en el sistema sobre las cuales se podría operar? y ¿Cuál es el régimen socio-técnico en el cual la innovación podría formar parte?

\section{La Gestión de residuos y el caso de estudio. Contexto general.}

La Agenda XXI (UNDS, 1992), en su Capítulo 21, Manejo Ecológicamente Racional de los Residuos Sólidos, establece las bases para un manejo integral de los residuos sólidos como parte del desarrollo sostenible. Por un lado, propone que dicho manejo debe contemplar la minimización de la producción de residuos, el reciclaje, la recolección y el tratamiento y disposición final adecuados; y por otro, deja en manos de cada país y ciudad el desarrollo de programas de acuerdo con sus características socioeconómicas y culturales.

De acuerdo con las metas de corto y mediano plazo fijadas en la Conferencia de Naciones Unidas sobre Medio Ambiente y Desarrollo (UNDS, 1992), para el año 2000, los países en 
desarrollo deberían haber establecido las capacidades para monitorear los procesos mencionados y los programas nacionales con metas propias para cada una de ellas.

En este contexto, Argentina desarrolló la Estrategia Nacional de Gestión de Residuos Sólidos Urbanos (ENGIRSU) en el año 2005 que se apoya se apoya en cuatro principios fundamentales 1) preservación de la salud pública; 2) preservación ambiental; 3) disminución significativa de residuos a generar, y 4) disposición con la aplicación de procesos de minimización, y valorización a través de las 4R (Reducción, Reúso, Reciclado, y Recompra) (SAyDS, 2005).

El Proyecto Nacional de Gestión Integral de Residuos Sólidos Urbanos (PNGIRSU) puesto en marcha por la Secretaría de Ambiente y Desarrollo Sustentable de la Nación (SAyDS) en 2006 tuvo, entre sus objetivos relacionados con la recuperación de residuos, la reinserción social de los trabajadores informales (SAyDS, 2011).

El modelo GIRSU que tiene como referente el modelo ISWM (Integrated Solid Waste Management) cuyo principal referente es ISWA (Integrated Solid Waste Asosiation) promueve con su modelo de gestión, el concepto de economía circular que involucra la integración de gestión de residuos con la cadena de valor bajo el concepto de la "cuna a la cuna" (cradle to cradle), proponiendo entre otras cuestiones para regiones como América Latina, la integración en el esquema del sector de la recolección informal y el cambio del modelo de negocio para empresas del rubro, pasando de ocuparse de la mera logística de RSU a su procesamiento como modo de generación de valor agregado.

Este modelo se ha venido desarrollando sobre diversos escenarios y contextos, incluyendo un gran número de experiencias fallidas en relación a la recuperación de reciclables y la incorporación de los recuperadores como estrategia de inclusión social (Gunsilius et al., 2011; Scheinberg, 2012), y ha tenido gran incidencia a nivel discursivo en los sistemas publicos de gestión de residuos.

Paralelamente la recolección y valorización de materiales en los residuos para la reinserción en las cadenas de valor de reciclables, tiene como conductor el valor económico intrínseco (latente o explícito) de los materiales y su potencial para volver a entrar en las cadenas productivas. En la mayoría de los países en vías de desarrollo, la cadena de valor de reciclables se es desarrollada por el sector privado (Scheinberg, Wilson and Rodic 2010; Scheinberg, Simpson and Gupt 2010; Scheinberg, 2015).

En el partido de General Pueyrredón (Ver Figura 1)pueden identificar en el tratamiento y disposición de residuos de Mar del Plata cuatro etapas (Gonzalez Insua y Ferraro, 2015) siendo la cuarta etapa en la que se implementa el Plan GIRSU sobre la cual se focaliza el caso de estudio.

La aplicación de GIRSU a nivel territorio en la incorporación de la gestión de materiales reciclables involucra estrategias de inclusión social que proponen la incorporación de los recuperadores informales que se encontraban en el Basural a Cielo Abierto (BCA) actualmente Playón de Contención. Al respecto establece que:

El sistema de gestión de residuos que se está impulsando en el municipio de General Pueyrredón atiende especialmente la cuestión social, ya que promueve la inclusión de los recuperadores que recolectaban materiales en el antiguo basural a cielo abierto, en nuevos esquemas de trabajo que no comprometan su 

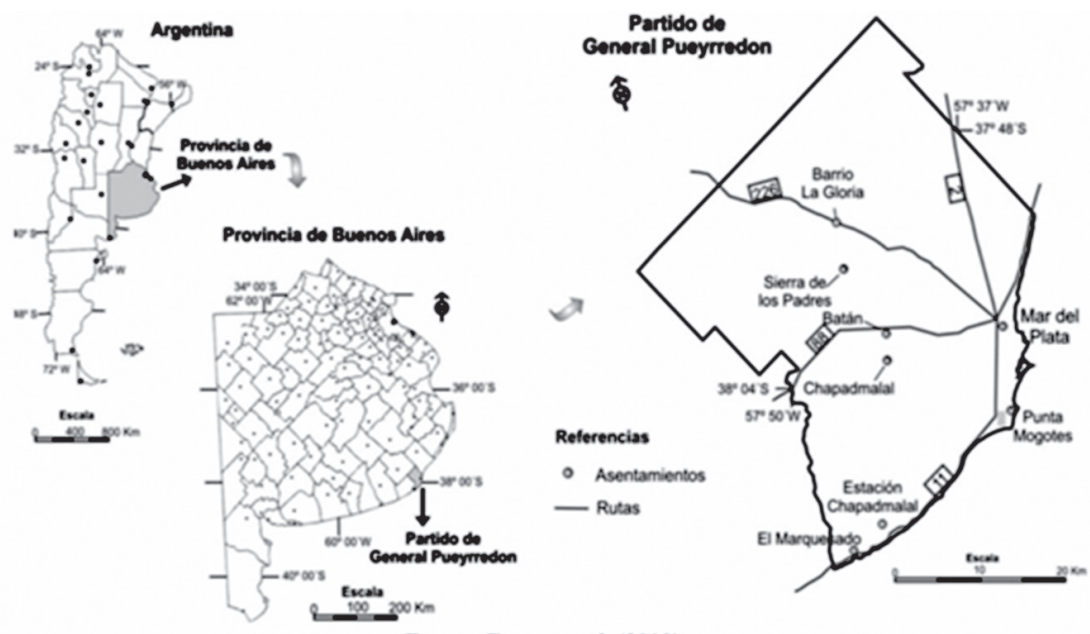

Figura 1. Localización del área de estudio. Fuente: Ferraro et al 2013.

salud y mejoren la situación de vulnerabilidad en que se encuentran inmersos desde hace casi dos décadas. (MGP, 2011, p. 51)

En el análisis de las dinámicas de organización de recuperadores en América Latina se sostiene que:

...la génesis de estos procesos de organización de recicladores reside en gran medida en la situación de vulnerabilidad e inestabilidad que caracteriza a esta población en nuestra región, lo que la lleva a buscar alternativas que le permitan mejorar sus condiciones de vida y de trabajo. Los procesos de organización tienen también su génesis en la toma de conciencia por parte de recicladores, gobiernos municipales, organizaciones de la sociedad civil y organismos multilaterales, de que la organización y "formalización” de esta población, mediante su inclusión en el sistema de manejo de RSM, es un camino viable para mejorar su situación laboral y para mitigar la vulnerabilidad e inestabilidad congénita que caracteriza a este grupo... (Terraza y Sturzenegger, 2010, p. 17) ${ }^{9}$

En este sentido y siguiendo la línea planteada por los autores mencionados, al hablar de Circuito Formal de recuperación y recuperadores formales, se alude a la "formalización" como parte del proceso de inclusión de los recuperadores en la estrategia GIRSU bajo la figura de Cooperativa de Trabajo. 
En la ciudad de Mar del Plata, el año 2012 se presenta el "Plan Básico Preliminar” de GIRSU con el que se inaugura el relleno sanitario en el Centro de Disposición Final (CDF) y se reacondiciona y reinaugura la Planta de Separación y Clasificación de Materiales (PSCM). De este modo, se inaugura oficialmente el relleno sanitario de la ciudad y se comienza con la implementación del Plan de separación de RSU. En este punto, se produce una modificación en la dinámica socio-técnica de la gestión de residuos municipal y se configura una nueva dinámica que involucra un Circuito Formal e Informal de reciclaje. Es sobre esta dinámica socio-técnica que se define la Situación Problema.

\section{La Situación- Problema en el funcionamiento de la gestión de recuperación de reciclables en Mar del Plata}

El proceso de construcción de la Situación-Problema involucra una aproximación desde una postura crítica-reflexiva, busca evidenciar las miradas de los grupos sociales relevantes, las agencias de los artefactos y las condiciones políticas del territorio (Thomas, Becerra, \& Davyt, 2013). Asimismo implica un grado de flexibilidad interpretativa en la identificación de los problemas (Thomas, 2009).

La construcción de la Situación-Problema y la identificación de las relaciones ProblemaSolución propuestas, bajo un análisis de flexibilidad interpretativa, proporciona conocimiento sobre cómo cada grupo social relevante entiende su problema, y por extensión la posible solución al mismo en base a sus conocimientos, saberes, condiciones materiales de entorno y ambiente percibidas, su historia, etc.

Si bien no es el objetivo de este trabajo la profundización en el análisis de la dinámica Problema-Solución, sino contribuir a la visualización de la complementariedad en el análisis sistémico interdisciplinar entre los marcos de TIS y PSS, a continuación se realiza un breve punteo de los Grupos Sociales Relevantes identificados en el territorio, y las tecnologías más relevantes en relación a la situación problema definida para este trabajo.

\subsection{Grupos Sociales Relevantes}

Municipalidad de General Pueyrredón (MGP). La Municipalidad de General Pueyrredón (MGP) es la responsable de la planificación e implementación de la GIRSU a nivel local. Asimismo, es quien administra los recursos públicos y actúa como organismo de control dentro del marco Normativo.

Banco Interamericano de Desarrollo (BID). El BID, como entidad financiera internacional financia la iniciativa de Ciudades Emergentes Sostenibles (ICES, 2010) en Mar del Plata. El financiamiento de BID proporcionó, entre otras cuestiones, los recursos necesarios para la reinauguración de la Planta Modelo como PSCM. 
Organismo Provincial para el Desarrollo Sostenible (OPDS). El Organismo Provincial para el Desarrollo Sostenible es la autoridad de aplicación en materia ambiental en el ámbito de la Provincia de Buenos Aires.

Cooperativa de trabajo Común Unión de Recuperadores Argentinos Ltda. (CURA Ltda.). La Cooperativa CURA Ltda., está conformada por recuperadores que desarrollaban sus actividades en el predio del basural del PGP. Desde mitad del 2012 con la reinauguración de la PSCM, efectúa el usufructo de la PSCM, como componente técnico-operativo de la GIRSU en un esquema de cogestión que implica una dinámica socio-técnica particular como parte de la estrategia de inclusión social. En la implementación de GIRSU representa un componente técnico y operativo de GIRSU.

Escuelas. Las escuelas son vehículo de conocimiento, a través de docentes y de los niños como portadores del mensaje difunden la necesidad de separación en origen, es un factor de sensibilización muy importante de la población. Asimismo las escuelas municipales forman parte del circuito de recolección de blancos y cartones.

Universidad. Registra programas de extensión y transferencia vinculados con la temática de la gestión de residuos desde una perspectiva socio-ambiental. Asimismo existen instituciones que se encuentran actualmente vinculadas con la problemática de estudio ( IHAM) y programas de extensión y Vinculación Socio Productiva (Vi.So.Pro) que acompañan a la cooperativa en el proceso de conformación y consolidación.

Federación Argentina de Cartoneros y Recicladores (FACyR). La Federación Argentina de Cartoneros y Recicladores nuclea a los trabajadores cartoneros, carreros, recicladores y recuperadores urbanos de todo el país, organizados en cooperativas u otras formas asociativas con el objetivo de defender sus derechos. FACyR es una de las organizaciones que componen la Confederación de Trabajadores de la Economía Popular (CTEP). Ambas han acompañado los reclamos de CURA Ltda. en relación a la co-gestión de la PSCM, así como el reclamo de los recuperadores en el playón de contingencia.

Confederación de Trabajadores de la Economía Popular (CTEP). La CTEP es una organización gremial, representativa de los trabajadores de la economía popular y sus familias. Se autodefine como una herramienta de lucha reivindicativa para la restitución de los derechos laborales y sociales que nos arrebató el neoliberalismo y que aún no hemos recuperado.

Recuperadores de playón contingencia. Los recuperadores del playón de contingencia son lo recuperadores del BCA que con la inauguración del relleno sanitario y las modificaciones, pasaron a recuperar al playón de contención. Son dentro de los recuperadores el GSR en mayor situación de vulnerabilidad.

Recuperadores “informales” en vía pública. Pueden ser definidos como aquellos que recorren las calles buscando entre los residuos eliminados por la población o a quienes se les entrega en mano, los elementos que reconocen como reciclables y por lo tanto vendibles, 
para obtener ingresos que les permitan subsistir. A diferencia de los GSR anteriores, no hay censo de esta población y se cuenta con escasa información o registro sobre los mismos en el territorio.

Empresa recolectora 9 de Julio. Empresa que realiza la recolección y transporte de los residuos en Mar del Plata desde 1992.

Acopiadores y galponeros informales. Dentro de este GSR podemos encontrar pequeños compradores/acopiadores barriales, y compradores móviles o los que esperan, comercializan por kg y a contado con los recuperadores informales de la vía pública (Barabino, 2014).

Acopiadores formales y grandes empresas recuperadoras. Dentro de este GSR están quienes adquieren o reciben materiales provenientes de la recolección, procediendo a su clasificación, acondicionamiento y compactado se encuentran bajo el registro formal de la Administración Federal de Ingresos Públicos (AFIP). Se registra una gran empresa recuperadora en la ciudad.

Empresas generadoras de scrap y grandes generadores. En este grupo encontramos Industrias, establecimientos comerciales o entidades administrativas que, como resultado de su actividad, generan residuos reciclables, generalmente tienen vínculos directos con acopiadores y es poco el volumen que se recupera mediante recuperadores informales.

Recicladoras. Definidas por la AFIP como establecimientos industriales que efectúan la transformación física, química o físico-química en su forma o esencia de los materiales a través de un proceso industrial, mediante la utilización de maquinarias o equipos, obteniendo de dicho proceso una materia prima o un nuevo producto. Son el último eslabón en la cadena de valor representado por grandes empresas industriales que elaboran productos específicos. Pueden ser empresas nacionales o multinacionales que pertenecen a Cadenas Globales de Valor (CGB) que ejercen poder y control sobre los proveedores, estableciendo los precios del mercado y los patrones de calidad de la materia prima (Caló, 2009).

\subsection{Tecnologías}

Teniendo en cuenta que la GIRSU funciona como una gran tecnología de la cual se derivan o alinean otras tecnologías, a continuación se enuncian las mismas diferenciando las que tecnologías involucradas directamente en GISRU y las que corresponden al circuito informal.

\section{Plan GIRSU-MDP}

- Plan de Separación en origen

- Sistema de recolección y transporte

- Licitación de Recolección y transporte RSU

- Circuito de recuperación de Blancos y Cartones.

- Recolección informal

- Acopio informal 


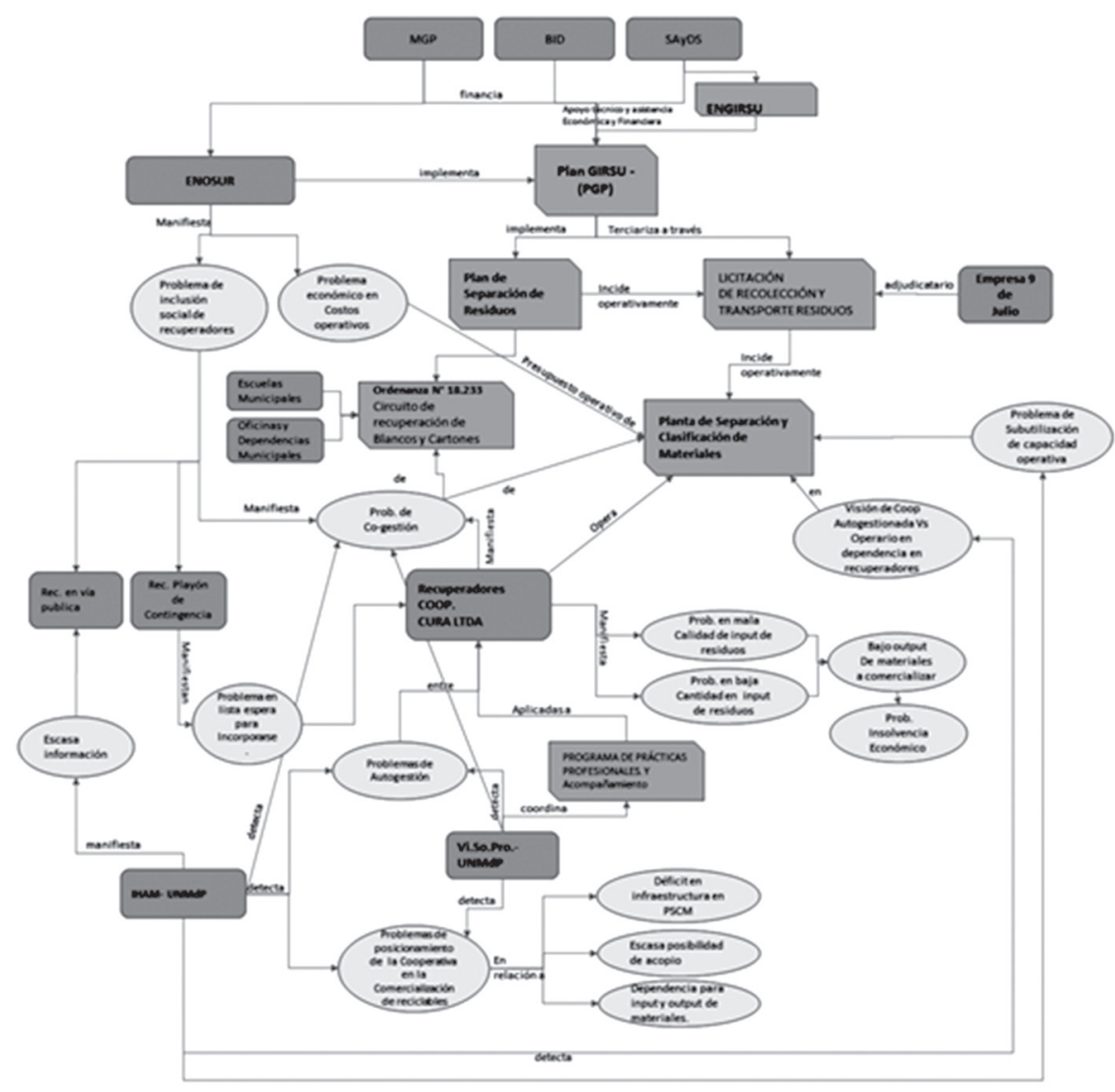

Figura 2. Situación Problema en el análisis del funcionamiento de la Gestión de recuperación de reciclables en Mar del Plata. Fuente: Elaboración propia.

\subsection{La Situación Problema en Mar del Plata.}

Como se puede ver en la Figura 2, la Situación-Problema presenta un sistema complejo que despliega diversas dinámicas socio-técnicas, entendidas y abordadas en este análisis como subsistemas (debido a la complejidad de las relaciones).

La flexibilidad interpretativa con la que se identifican los problemas manifestados por los actores en el territorio en relación al funcionamiento de la Gestión de recuperación de reciclables en Mar del Plata, permite evidenciar las racionalidades dentro de los actores frente a un mismo artefacto como por ejemplo, en la PSCM (como artefacto y tecnología dentro de la GIRSU). Asimismo, permite comprender como conceptualizan los problemas 
los diferentes actores y como podrían interpretar y reaccionar frente a una determinada intervención.

Si se toma como ejemplo la PSCM como artefacto y tecnología dentro de la GIRSU, podemos aproximarnos a como conciben los problemas entorno a este artefacto los GSR.

- La Municipalidad plantea problemas relacionados con la inclusión social de los recuperadores (la planta no cumple con el objetivo cuantitativo de recuperadores a incorporar en la PSCM), problemas económicos (evidenciados como presupuesto de mantenimiento y operación de la planta) y problemas de cogestión de la planta con la Cooperativa bajo el formato de convenio de cooperación en el cual operan.

- La Cooperativa que opera bajo una lógica de comercialización de reciclables, manifiesta problemas en la mala calidad de input de residuos (en referencia a la mezcla de los materiales reciclables con húmedos), baja cantidad de residuos que ingresan a la planta (en relación a la cantidad de residuos sobre los cuales hacen segunda separación y comercializan y las ganancias derivadas de la misma), insolvencia económica (debido a las ganancias de la comercialización de reciclables), problemas con el mantenimiento de las instalaciones (los tiempos administrativos para la reparación de la infraestructura no acompañan las necesidades económicas, si la planta no opera la cooperativa no cobra), problemas de cogestión, etc.

- Desde el ámbito universitario, tanto IHAM como Vi.So.Pro manifiestan problemas de autogestión de la cooperativa (visibilizan conflictos de conformación y consolidación de la cooperativa como tal), problemas de posicionamiento de la Cooperativa en la Cadena de Valor que a su vez se relacionan con problemas de déficit de la infraestructura, escasa posibilidad de acopio y dependencia para el ingreso y egreso de materiales a la PSCM.

- Finalmente desde los recuperadores del Playón la problemática en relación a la PSCM es que los mismos se encuentran en una lista de espera para la incorporación en la operación de la planta con la cooperativa, y con el ingreso que genera la cooperativa no admite incorporación de más asociados.

$\mathrm{Al}$ analizar el funcionamiento de la gestión de recuperación de reciclables a partir de lo identificado en el gráfico de la Situación Problema, y con el objetivo de comprender las múltiples aristas se definieron como paso siguiente 5 dinámicas Problema-Solución a analizar que responden en su orden a una estrategia de visualización desde lo macro a lo micro que acompaña la lectura sistémica. Estas dinámicas correspondieron a: 1) GIRSUMDP; 2) Cadena de Valor de reciclables, 3) Dispositivo PSCM; 4) Recuperadores Formales e Informales y 5) Cooperativa CURA Ltda. 


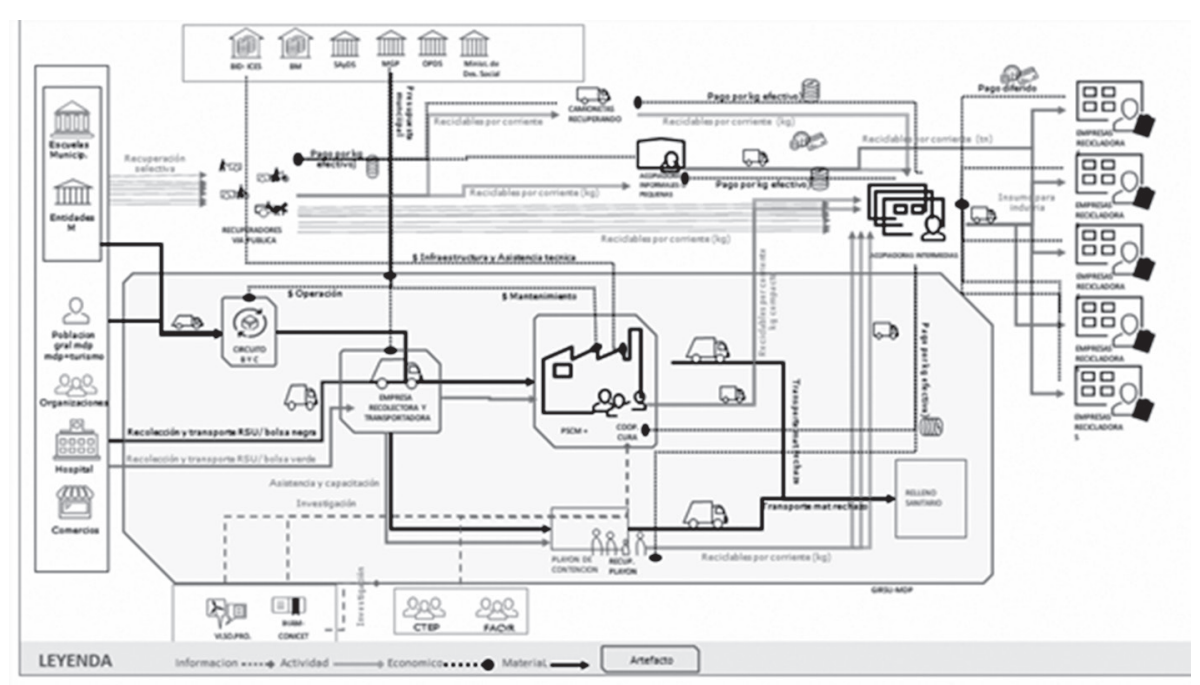

Figura 3. Mapa sistémico de la recuperación de reciclables en Mar del Plata. Fuente: Elaboración propia.

\section{Aportes desde Producto-Sistema-Servicio. El mapa sistémico}

El mapa sistémico como herramienta permite visualizar la estructura del sistema, los actores y las respectivas interacciones y puede ser utilizado tanto en la fase de análisis estratégico como en el diseño de estrategias sistémicas. Constituye básicamente una representación gráfica que visualiza a los actores o grupos sociales relevantes involucrados en el sistema (tanto primario como secundario) y las diferentes interacciones entre estos actores focalizando en los flujos materiales, de información, monetarios o de trabajo.

De esta manera el Mapa Sistémico como herramienta de representación es definida (Vezolli et al, 2014) como un codificada y progresiva. Constituye un sistema codificado ya que es un "dibujo técnico" de los actores que permite representar alternativas sistémicas en un formato reproducible y comparable. Al mismo tiempo es progresivo en el sentido que a medida que se profundiza en el análisis o la propuesta de diseño, se vuelve más específico. El Mapa Sistémico en interrelación con el análisis de TIS permite profundizar sobre las dinámicas socio-técnicas existentes dentro del sistema y asimismo visualizar las alianzas socio-técnicas que se construyen para el funcionamiento del mismo desde un marco general en donde se vinculan los flujos de los materiales, de información, económicos, los principales GSR y artefactos.

De igual manera, permite ver las relaciones entre los componentes de GIRSU como artefacto en un sistema técnico-operativo de gestión de residuos reciclables en conjunto con el mercado que incorpora los reciclables en la Cadena de Valor. 
La Figura 3 visualiza por un lado los componentes del sistema GIRSU como artefacto y tecnología en relación a los reciclables junto con los GSR relevantes, directa e indirectamente vinculados, diferenciando en una primera instancia los que se encuentran dentro de GIRSU como tecnología de gestión de residuos e inclusión social, de los que se encuentran por fuera de la aplicación directa de la misma.

Hacia adentro del artefacto GIRSU podemos ver otros tres que son descriptos con mayor detalle más avanzado el trabajo; el circuito de blancos y cartones (ByC), la licitación del servicio de higiene y recolección (que dentro de la misma incorpora a su vez al circuito de ByC) pero en este caso se diferencia para hacer alusión a la recolección y transporte de los residuos sólidos urbanos, y el convenio de cogestión ENOSUR-CURA Ltda. en la operación de la PSCM. Esta estructura y vinculación esta encadenada principalmente siguiendo los flujos de los RSU, no obstante se pueden ver algunas de las relaciones económicas y de información dentro del esquema.

Por otro lado y fuera de la incumbencia directa de GIRSU se puede ver a los recuperadores informales de la vía pública, como GSR relevante que configura el primer eslabón en el circuito de recolección de reciclables, y como se encadenan con los siguientes GSR relevantes que configuran la CDV como son los acopiadores e intermediarios informales, luego formales y las empresas recicladoras. Estas últimas no se encuentran en el territorio de Mar del Plata, sin embargo se las menciona ya que son quienes ponen los parámetros por sobre los que operan los eslabones anteriores.

\section{Conclusiones}

Debido a que lo presentado corresponde a una investigación que se encuentra en desarrollo, no es posible presentar en este artículo los mapas sistémicos resultantes a partir de la identificación de los cinco problemas-solución derivados de la Situación-Problema, ni los nudos críticos del sistema con sus respectivas propuestas innovativas. Sin embargo es posible verificar que el Mapa Sistémico como herramientas de PSS aplicada en el análisis estratégico, contribuye a ordenar y visualizar las relaciones entre los actores, artefactos, tecnologías e instituciones, así como los flujos información, material, económicos, etc.

La aproximación desde el marco socio-técnico contribuye a identificar los actores y GSR de manera que no solo identifica a los actores directamente relacionados con el funcionamiento de la gestión de reciclables como componentes operativos, sino que contribuye a la identificación de actores relevantes del ámbito universitario, instituciones, centros de investigación o diferentes organizaciones, es decir una visibilizar a la red heterogénea de actores que tienen relevancia significativa a nivel social, económico, político y cultural.

El análisis de la Situación-Problema permitió a su vez, visibilizar como conciben el funcionamiento de la gestión de reciclables los GSR que componen la red, así como identificar las racionalidades de los mismos.

$\mathrm{Al}$ igual que sucede con la eco-eficiencia del sistema, a nivel propositivo no todos los PSS resultaran sustentables, inclusivos o eco-eficientes, sin embargo, la implementación de las herramientas de PSS como parte del desarrollo de capacidades diseño estratégico puede contribuir a la generación de relaciones económico-productivas inclusivas, acceso a bienes 
y generación de empleo si en el análisis sistémico las mismas constituyen los requerimientos del sistema.

Finamente, para poder realizar intervenciones sistémicas, es fundamental comprender las problemáticas, expectativas, las capacidades de negociación y alineación de los actores así como a organización de las mismas dentro del territorio y la aproximación metodológica resulto complementaria.

\section{Notas}

1. SusHouse: Strategies towards the Sustainable Household (EU funded, 1998-2000) (see Vergragt 2002).

2. ProSecCo: Product-Service Co-design (EU funded, 2002-2004)

3. HiCS: Highly Customerised Solutions (EU funded, 2001-2004) (see Manzini, Collina and Evans 2004).

4. MEPSS: MEthodology for Product Service System development (EU funded, 20022005) (see Van Halen, Vezzoli and Wimmer 2005).

5. SusProNet: Sustainable Product-Service co-design Network (EU funded, 2002-2005) (see Tukker and Tischner 2006).

6. Vezzoli, C., Kohtala, C., \& Srinivasan, A. (2014). Product-Service System Design for Sustainability. http://doi.org/http://dx.doi.org/10.9774/GLEAF.978-1-909493-69-8_8.

7. El "funcionamiento" o "no-funcionamiento" (Bijker, 1995) de un artefacto es resultado de un proceso de construcción socio-técnica en el que intervienen, normalmente de forma auto-organizada, elementos heterogéneos (materiales, sistemas, conocimientos, regulaciones, financiamiento, prestaciones, etc.). Es una contingencia que se construye social, tecnológica y culturalmente y supone complejos procesos de adecuación de respuestas/ soluciones tecnológicas a concretas y particulares articulaciones socio- técnicas históricamente situadas (Thomas, 2009).

8. Una dinámica socio-técnica es un conjunto de patrones de interacción de tecnologías, instituciones, políticas, racionalidades y formas de constitución ideológica de los actores [...]. Este concepto sistémico sincrónico permite insertar una forma determinada de cambio socio-técnico (una serie de artefactos, una trayectoria socio-técnica, una forma de relaciones problema-solución, por ejemplo) en un mapa de interacciones (Thomas, 2008, p. 249).

9. Nota Técnica que forma parte de los productos de conocimiento generados por la iniciativa de Agua y Saneamiento, aprobada por el Directorio del Banco Interamericano de Desarrollo en mayo de 2007 y publicada en el 2010.

\section{Referencias}

Barabino, N. (2015). “La importancia de la recolección informal de residuos urbanos reciclables: Análisis de los aspectos sociales, ambientales y económicos: La situación en la ciudad de Mar del Plata”. Tesis de Maestría, Universidad Nacional de Mar del Plata, Argentina. 
Caló, J. (2009). La cadena de valor del reciclado de residuos sólidos urbanos .La dinámica sociotécnica de los procesos de reciclado en las cooperativas de recuperadores urbanos surgidas a partir de los cambios económicos, sociales y tecnológicos de la crisis del 20. Universidad Nacional de Quilmes. Disponible en: http://www.proyectaryproducir.com.ar/?page_id=16

Carenzo, S.; Acevedo, R. y Barbaro, J. (2013). “Construyendo oficio: experiencias laborales de integrantes de una Planta Social de Separación en el CEAMSE”. Trabajo y Sociedad Sociología Del Trabajo - Estudios Culturales - Narrativas Sociológicas y Literarias, 20, 221-238.

Ehrenfeld, J. R. (2008). Sustainability by Design: A Subversive Strategy for Transforming Our Consumer Culture (Yale University Press).

Ferraro, R.; Zulaica, L. y Echechuri, H. (2013). "Perspectivas de abordaje y caracterización del periurbano de Mar del Plata, Argentina”, en Letras Verdes, Revista del Programa de Estudios Socioambientales, FLACSO, Ecuador, No 13, pp. 19-40. Disponible en: http:// revistas.flacsoandes.edu.ec/letrasverdes/article/view/926/887

Gonzalez Insua, M., \& Ferraro, R. (2015). Los residuos sólidos urbanos en Mar del Plata, Argentina iproblemática ambiental o insumos para la industria? Letras Verdes. Revista Latinoamericana de Estudios Socioambientales, 17(17), 57-85. Disponible en: http://doi. org/10.17141/letrasverdes.17.2015.1446

Gunsilius, E.; Spies, S.; García-Cortes, S.; Medina, M.; Dias, S.; Scheinberg, A. y Ruiz, S. (2011). Recovering resources, creating opportunities. Integrating the informal sector into Solid Waste Managment. Disponible en: http://scholar.google.com/scholar?hl=en\&btn $\mathrm{G}=$ Search\&q=intitle:Recovering+resources+Creating+opportunities.+Integrating+the +informal+sector+into+Solid+Waste+Managment\#0\%5Cnhttp://scholar.google.com/ scholar?hl=en\&btnG=Search\&q=intitle:Recovering+Resources+Cre.

Paiva, V. (2004). "Las cooperativas de recuperadores y la gestión de residuos sólidos urbanos en el área metropolitana de Buenos Aires". Revista Theomai. Disponible en: http:// revista- theomai.unq.edu.ar/numespecial2004/artpaivanumespec2004.htm.

Paiva, V. (2005). "Modos formales e informales de recolección y tratamiento de los residuos. Ciudad de Buenos Aires. Siglos XVI al XX”, 150. Disponible en: http://www.iaa.fadu.uba. ar/publicaciones/critica/0150.pdf

Paiva, V., y Perelman, M. (2008). "Recolección Y Recuperación Informal De Residuos. La Perspectiva De La Teoría Ambiental Y De Las Políticas Públicas". Ciudad De Buenos Aires 2001-2007. Cuaderno Urbano 7 - Espacio, Cultura Y Sociedad, 7, 35-54.

Scheinberg, A. (2012). Informal Sector Integration and High Performance Recycling: Evidence from 20 Cities. WIEGO Working Paper (Vol. 23).

SAyDS (Secretaría de Ambiente y Desarrollo Sustentable) (2005). "Estrategia Nacional para la Gestión Integral de Residuos Sólidos Urbanos”. Buenos Aires. Argentina.

SAyDS (Secretaria de Ambiente y Desarrollo Sustentable). Observatorio Nacional para la Gestión de Residuos Sólidos Urbanos (2009). “Informe Final Septiembre 2009”. Argentina.

Schamber, P. J. y Suárez, F. (2006). "Actores sociales y cirujeo y gestión de residuos. Una mirada sobre el circuito informal del reciclaje en el conurbano bonaerense"- Artículos de Realidad Económica - Noticias, Artículos Especiales y Dossiers. IADE - Realidad Económica, 190, 14.

Thomas, H. (2009). Tecnologías para la inclusión social y políticas públicas en América Latina. Grupo de Estudios Sociales de la Tecnología y la Innovación, (June), 1-32. 
Thomas, H. (2008): Estructuras cerradas vs. procesos dinámicos: trayectorias y estilos de innovación y cambio tecnológico, en Thomas, H. y Buch, A. (eds.), Actos, actores y artefactos. Sociología de la tecnología. Bernal: Editorial de la UNQ, pp. 63-100

Thomas, H.; Becerra, L. \& Davyt, A. (2013). Repensar el desarrollo y el cambio tecnológico. De la crítica conceptual a la propuesta normativa. Conferencia Internacional LALICS 2013 "Sistemas Nacionales de Innovación y Políticas de CTI para un Desarrollo Inclusivo y Sustentable", (June 2017), 23. Disponible en: http://www.redesist.ie.ufrj.br/ lalics/papers/131_Repensar_el_desarrollo_y_el_cambio_tecnologico_De_la_critica_ conceptual_a_la_propuestas_normativa.pdf

UNDS (United Nations Division for Sustainable Development) (1992). United Nations Conference on Environment \& Development Rio de Janeiro, Brazil, 3 to 14 June 1992. Agenda 21.

UNEP (2002) Product-Service Systems and Sustainability: Opportunities for Sustainable Solu- tions (Paris: United Nations Environment Programme, Division of Technology Industry and Economics, Production and Consumption Branch).

UNEP (2009) Design for sustainability. A step-by-step approach (Paris, France: UNEP).

Vezzoli, C.; Kohtala, C. \& Srinivasan, A. (2014). Product-Service System Design for Sustainability. Disponible en: http://doi.org/http://dx.doi.org/10.9774/GLEAF.978-1-909493-69-8_8.

\begin{abstract}
Initially, the approach of Sustainable Design in Argentina was dominated by recycling practices, solving problems towards the end of life of the products. Subsequently, the notion of Life Cycle allowed to expand the limits of analysis to account for the impact of design decisions in all stages. While both approaches have been useful, in the field of design management these views are limited, because in practice they converge more than just objects: the actors, the organizations and their multiple relationships. Adhering to the criteria of sustainability, it is worth asking what are the tools and methodologies that would be necessary for a comprehensive approach to the problems in Design Management. Tools that allow ordering information and also serve as a bridge for the joint presentation of problems with the actors in the territory.

The PSS approach abandons the traditional conception of design, development and manufacture of products to be placed in solutions where the generation of value is separated from the consumption of resources and energy (Vezzoli et al, 2014). In this way the innovations are placed in the design of services and relationships between the actors., From their practical application in a case study in the strategic analysis phase.

Technologies for Social Inclusion (TIS) in the same line, are aimed at the generation of capabilities to solve systemic problems, and the generation of local dynamics of production, technological change and socio-technically appropriate innovation that benefit everyone (Thomas et al, 2012).

In this line, this article reviews the notions and tools proposed by two theoretical frameworks, on one hand the Product-System-Service (PSS) approach and on the other the Social Inclusion Technologies with the aim of contributing to the perspective of the urban sustainability approaches based on an interdisciplinary approach. The analysis of the
\end{abstract}


Formal Circuit of urban solid waste recovery of Mar del Plata city is taken as a case study, analyzing on the one hand the "Situation-Problem" that determines the current system status from the Social Inclusion Technologies framework, and on the other hand, the application of the Systemic Map as a complementary methodological tool in the analysis.

In strategic management and systemic approaches, it is essential to understand the problems, expectations, negotiation and alignment capacities of the actors as well as their organization within the territory. The methodological approach has been complementary, allowing through the TIS framework the visibility of the heterogeneous network of actors of significant relevance at a social, economic, political and cultural level, and how each Relevant Social Group belonging to this network conceives the problems in relation to the object of study.

Likewise, the Systemic Map as a PSS tool applied in the strategic analysis, contributes to organize and visualize the relationships between actors, artifacts, technologies and institutions, as well as information, material, economic flows, etc.

Key words: Product-System-Service - Technologies of Social Inclusion - Recyclable Urban Solid Waste - Design for Sustainability - Urban Sustainability.

Resumo: Inicialmente, a abordagem do Design Sustentável na Argentina foi dominada por práticas de reciclagem, resolvendo problemas até o fim da vida dos produtos. Posteriormente, a noção de ciclo de vida permitiu expandir os limites de análise para explicar o impacto das decisões de design em todas as etapas. Embora ambas as abordagens tenham sido úteis, no campo do gerenciamento de design essas visualizações são limitadas, porque, na prática, elas convergem mais do que apenas objetos: os atores, as organizações e seus múltiplos relacionamentos. Aderindo aos critérios de sustentabilidade, é possível perguntar quais são as ferramentas e metodologias necessárias para uma abordagem abrangente dos problemas na gestão do Design. Ferramentas que permitem informações de pedidos e também servem de ponte para a apresentação conjunta de problemas com os atores no território.

A abordagem PSS abandona a concepção tradicional de design, desenvolvimento e fabricação de produtos a serem colocados em soluções onde a geração de valor é separada do consumo de recursos e energia (Vezzoli et al, 2014). Desta forma, as inovações são colocadas no design de serviços e relações entre os atores, a partir de sua aplicação prática em um estudo de caso na fase de análise estratégica.

As Tecnologias para a Inclusão Social (TIS) na mesma linha visam a geração de capacidades para resolver problemas sistêmicos e a geração de dinâmicas locais de produção, mudanças tecnológicas e inovações socio-tecnicamente apropriadas que beneficiam a todos (Thomas et al, 2012).

Nesta linha, este artigo analisa as noções e ferramentas propostas por duas estruturas teóricas, por um lado, a abordagem do Produto-Sistema-Serviço (PSS) e, por outro lado, as Tecnologias de Inclusão Social com o objetivo de contribuir para a perspectiva da abordagem de sustentabilidade urbana com base em uma abordagem interdisciplinar. A análise do Circuito Formal de recuperação de resíduos sólidos urbanos de Mar del Plata é tomada como um estudo de caso, analisando, por um lado, o «Problema de Situação» que determina o sta- 
tus atual do sistema a partir da estrutura de Tecnologias de Inclusão Social e Por outro lado, a aplicação do Mapa Sistêmico como ferramenta metodológica complementar na análise. Na gestão estratégica e nas abordagens sistêmicas, é essencial compreender os problemas, as expectativas, a negociação e as capacidades de alinhamento dos atores, bem como sua organização dentro do território. A abordagem metodológica tem sido complementar, permitindo, através do quadro TIS, a visibilidade da rede heterogênea de atores de relevância significativa a nível social, econômico, político e cultural e como cada Grupo Social Relevante pertencente a esta rede concebe os problemas em relação para o objeto de estudo.

Do mesmo modo, o Mapa Sistêmico como ferramenta PSS aplicada na análise estratégica, contribui para organizar e visualizar as relações entre atores, artefatos, tecnologias e instituições, bem como informações, materiais, fluxos econômicos, etc.

Palavras chave: Produto-Sistema-Serviço, Tecnologias de Inclusão Social, Resíduos Sólidos Urbanos Recicláveis, Design para a Sustentabilidade, Sustentabilidade Urbana. 